Tatiana B Cereija, Joana Castro, Patrícia Alves, Nuno Cerca

\title{
Influence of anaerobic conditions on vaginal microbiota recovery from bacterial vaginosis patients
}

Institute for Biotechnology and Bioengineering (IBB), Centre for Biological Engineering, University of Minho, 4710-057 Braga, Portugal

${ }^{*}$ Corresponding author: email: nunocerca@ceb.uminho.pt, Phone: (+351) 253604423, Fax (+351) 253604429

Bacterial vaginosis (BV) is one of the most common infections in women of reproductive age. Clinical studies have shown an association among BV and abnormal pregnancy, pelvic inflammatory disease and increased risk of sexually transmitted infections, including HIV. This disorder was first described in 1914 by Curtis as a "white discharge" syndrome and despite the decades of research we have only limited, and clearly not conclusive, evidence of microbial cause of BV, mechanism of disease and effective treatment.

The development of molecular techniques such as Denaturing Gradient Gel Electrophoresis (DGGE) and DNA sequencing produced a clearer picture of the complexities of the vaginal microbiota. It has also become more apparent that none of the microorganisms already isolated from the vagina are likely to be the sole pathogen responsible for BV. Thus, improved knowledge of the relationship between different species of bacteria and their host is critical to a better understanding of both women's health and illness. In order to comprehend the dynamic interaction between bacteria-bacteria and bacteria-host it becomes necessary to isolate bacteria from vaginal samples and to use them in in vitro and in vivo assays. The human vagina has a very specific environment regarding $\mathrm{pH}$, nutrient availability and oxygen tension, being populated by a large range of bacteria from facultative to obligated anaerobic species. To isolate bacteria from such a complex niche, rich media should be used in order to promote the growth of different species. By using Columbia Agar (CBA) with $5 \%$ horse blood and by incubating at $37^{\circ} \mathrm{C}$ in the presence of $5 \%, 10 \% \mathrm{CO}$ or in anaerobic condition generated by AnaeroGen $^{\mathrm{TM}}$ (Oxoid), we were able to recover different bacteria species, from each of the anaerobic conditions tested, from BV vaginal samples, including some species never reported, as far as we know, such as Brevibacterium ravenspurgense, Corynebacterium tuscaniense, Klebsiella variicola, Nosocomiicoccus ampullae, Staphylococcus warneri and Bacillus firmus.

Despite the development of molecular methods and their unquestionable advantages in characterising the vaginal microbiota, classical bacteriology will nevertheless be required to elucidate the etiology of BV since the isolation of the pathogenic agent will be always required. Our findings revealed that slight differences in anaerobic conditions were crucial for the isolation of novel BV-associated organisms. Further in vitro studies of all the isolated bacteria will promote a better understanding of the dynamics of their interaction and will potentially reveal how BV develops and influences other sexual transmitted diseases.

\section{Acknowledgements}

This work was supported by European Union funds (FEDER/COMPETE) and by national funds (FCT) under the project with reference FCOMP-01-0124-FEDER-008991 (PTDC/BIAMIC/098228/2008). 


\section{Competing interests}

None.

\section{References}

[1] Guédou FA, Damme LV, Mirembe F, et al. Intermediate vaginal flora is associated with HIV prevalence as strongly as bacterial vaginosis in a cross-sectional study of participants screened for a randomised controlled trial. Sex Transm Infect 2012;88:545-51.

[2] Curtis $\mathrm{AH}$. On the etiology and bacteriology of leucorrhoea. Surg Gynecol Obstet 1914;18:299-306.

[3] Martin $\mathrm{DH}$. The microbiota of the vagina and its influence on women's health and disease. Am J Med Sci 2012;343:2-9.

[4] Cribby S, Taylor M, Reid G. Vaginal microbiota and the use of probiotics. Interdiscip Perspect Infect Dis 2008;2008:256490. 